\title{
ESTADO DE SAÚDE E REPRESENTAÇÕES SOBRE A DOENÇA NA PERSPECTIVA DE PORTADORES DE DIABETES MELLITUS
}

\section{HEALTH STATE AND REPRESENTATIONS ABOUT THE DISEASE FROM THE PERSPECTIVE OF PATIENTS WITH DIABETES MELLITUS}

\section{EL ESTADO DE LA SALUD Y LAS REPRESENTACIONES ACERCA DE LA ENFERMEDAD DESDE LA PERSPECTIVA DE PACIENTES CON DIABETES MELLITUS}

\author{
Luciana Soares Costa Santos ${ }^{1}$ \\ Alessandra Thomaz de Andrade ${ }^{2}$ \\ Fernanda Machado Silva-Rodrigues ${ }^{3}$ \\ Livia Keismanas de Ávila ${ }^{4}$
}

\begin{abstract}
Como citar este artigo: Santos LSC, Andrade AT, Silva-Rodrigues FM, Ávila LK. Estado de saúde e representações sobre a doença na perspectiva de portadores de Diabetes Mellitus. Rev baiana enferm. 2021(35):e42071.

Objetivo: avaliar o estado de saúde e suas dimensões em pacientes com Diabetes Mellitus, atendidos em um hospital de ensino, à luz do Modelo do Senso Comum. Método: estudo descritivo, com análise qualitativa dos dados. Participaram 20 pacientes hospitalizados e em atendimento ambulatorial. Os dados foram submetidos à análise de conteúdo e interpretados segundo os conceitos do Modelo do Senso Comum. Resultados: os discursos foram categorizados de acordo com a identidade da doença e suas concepções; duração e tempo para o diagnóstico e os sintomas; causas da doença e suas explicações; consequências e tratamento; controle e percepções sobre o prognóstico. Considerações finais: as concepções do estado de saúde e suas dimensões relacionadas ao Diabetes Mellitus, interpretadas à luz do Modelo do Senso Comum, descreveram-no como uma doença com sinais físicos e com potencial de complicação, inclusive associada à morte; contudo, também revelaram o desconhecimento das reais causas e consequências da doença.
\end{abstract}

Descritores: Concepções. Diabetes Mellitus. Processo Saúde-Doença.

Objective: evaluating the health status and its dimensions in patients with Diabetes Mellitus treated in a teaching hospital in the light of the Commonsense Model. Method: a descriptive study with qualitative analysis of the data. Participated twenty patients hospitalized and in outpatient care. The data were submitted to content analysis and interpreted according to the concepts of the Commonsense Model. Results: the discourses were categorized according to the identity of the disease and its concepts; duration and time for diagnosis and symptoms; causes of the disease and its explanations; consequences and treatment; control and perceptions about prognosis. Final considerations: the concepts of health status and its dimensions related to Diabetes Mellitus interpreted in the light of the Commonsense

Enfermeira. Doutora em Ciências. Docente da Faculdade de Ciências Médicas da Santa Casa de São Paulo. São Paulo, São Paulo, Brasil. luciana.santos@fcmsantacasasp.edu.br. http://orcid.org/0000-000।-5708-1460.

Enfermeira. Pesquisadora independente. São Paulo, São Paulo, Brasil. http://orcid.org/0000-000 I-9558-42 I0.

Enfermeira. Doutora em Ciências. Docente na Faculdade de Ciências Médicas da Santa Casa de São Paulo. São Paulo, São Paulo, Brasil. http://orcid.org/0000-0002$8412-2333$.

4 Enfermeira. Doutora em Enfermagem. Docente na Faculdade de Ciências Médicas da Santa Casa de São Paulo. São Paulo, São Paulo, Brasil. http://orcid.org/ $0000-0001-8739-1398$ 
Model described it as a disease with physical signs and potential for complication, even associated with death; however, they also revealed ignorance of the real causes and consequences of the disease.

\section{Descriptors: Hypertension. Income. Health Economics. Eating Behavior.}

Objetivo: evaluar el estado de salud y sus dimensiones en pacientes con Diabetes Mellitus tratados en un hospital docente a la luz del Modelo de Sentido Común. Método: es un estudio descriptivo con análisis cualitativo de los datos. Participaron veinte pacientes hospitalizados y en atención ambulatoria. Los datos fueron sometidos al análisis de contenido e interpretados de acuerdo con los conceptos del Modelo de Sentido Común. Resultados: los discursos fueron categorizados de acuerdo con la identidad de la enfermedad y sus conceptos; duración y tiempo para el diagnóstico y los síntomas; causas de la enfermedad y sus explicaciones; consecuencias y tratamiento; control y percepciones acerca del pronóstico. Consideraciones finales: los conceptos del estado de salud y sus dimensiones relacionadas con la Diabetes Mellitus, interpretadas a la luz del Modelo de Sentido Común, la describieron como una enfermedad con signos físicos y potencial de complicación, incluso asociada con la muerte; sin embargo, también revelaron ignorancia de las causas y consecuencias reales de la enfermedad.

Descriptores: Hipertensión. Renta. Economía de la Salud. Comportamiento Alimenticio.

\section{Introdução}

O impacto das doenças crônicas não transmissíveis (DCNT) vem superando a dimensão física e afetando de forma negativa o indivíduo, abrupta ou cronicamente. Um dos cenários de saúde que vem suscitando inúmeras preocupações, devido às alterações física, emocional e social refere-se ao Diabetes Mellitus (DM) ${ }^{(1)}$.

O DM consiste em um distúrbio metabólico caracterizado por hiperglicemia constante, decorrente da deficiência na produção de insulina ou em sua ação, ou por ambos os mecanismos. Atualmente estima-se que 463 milhões de pessoas sejam portadoras de DM em todo o mundo ${ }^{(1)}$. A hiperglicemia constante está associada a complicações crônicas micro e macrovasculares, além do aumento da morbidade, da redução da qualidade de vida (QV) e da elevação da taxa de mortalidade $^{(2)}$.

As complicações relacionadas ao DM incluem a hipoglicemia, a cetoacidose diabética (CAD) e o estado hiperglicêmico hiperosmolar (EHH). A hiperglicemia contribui para complicações macrovasculares que causam doença cerebrovascular, doença arterial coronariana e doença vascular periférica, enquanto as microvasculares causam retinopatia, nefropatia e neuropatia, e essas são especificas de indivíduos diabéticos ${ }^{(3)}$.

Observa-se aumento da prevalência do DM associado a diferentes fatores que incluem a modificação dos centros urbanos, transição epidemiológica, mudanças nutricionais, estilo de vida, sedentarismo, sobrepeso ou obesidade, aumento populacional e envelhecimento, além da maior sobrevida da população ${ }^{(2)}$.

Segundo a Organização Mundial de Saúde (OMS), a glicemia aumentada é o terceiro fator associado à mortalidade precoce, só perdendo para a incidência da hipertensão arterial sistêmica (HAS) e o tabagismo, tornando-se um problema de saúde pública, que implica em medidas de controle para o DM e suas complicações ${ }^{(2)}$.

A Associação Americana de Diabetes (ADA) destaca que é observada uma combinação de fatores que incluem o baixo desempenho dos sistemas de saúde, pouco conhecimento da população sobre DM em relação ao início súbito dos sintomas ou progressão do DM tipo 2, condição que pode permanecer indetectada por vários anos, dando oportunidade ao desenvolvimento das complicações. Dados apontam que aproximadamente 50\% dos casos de DM nos adultos não são diagnosticados e que 84,3\% de todos os casos de diabetes não diagnosticados ocorrem em países em desenvolvimento ${ }^{(4)}$.

Outro aspecto importante relacionado ao DM está associado ao aumento de hospitalizações, à incidência de doenças cardiovasculares e cerebrovasculares, alterações visuais, renais 
e complicações vasculares que levam a amputações, principalmente em membros inferiores, necessitando maior utilização dos serviços de saúde, o que futuramente poderá refletir em altos custos nos sistemas de todos os países, independentemente do seu desenvolvimento econômico ${ }^{(4)}$.

Estudo realizado com pacientes diabéticos considerou que a qualidade de vida refere-se à percepção do bem-estar e os achados podem subsidiar informações para o cuidado de pessoas com DM, permitindo um planejamento mais apropriado $^{(5)}$.

As doenças crônicas, como o DM, tendem a incorporar características fundamentadas nas crenças de seus portadores, o que pode comprometer a aceitação da doença e o seu tratamento. Entender as concepções dos indivíduos acerca das alterações em seu estado de saúde pode melhor contextualizar intervenções voltadas ao controle de determinadas patologias, como o DM. Assim, para o Modelo de Autorregulação do Comportamento em Saúde ou Modelo do Senso Comum (MSC), o estado de saúde representa a integridade e o bem-estar do indivíduo quando este não se sente ameaçado pelos acontecimentos negativos da doença que o acomete ${ }^{(6)}$.

Segundo o MSC, a percepção da doença pode ser compreendida em cinco dimensões: identidade (expressa por rótulos abstratos, como o nome da doença e seus sintomas associados); causas (fatores relacionados à origem da doença); duração (curso da doença: aguda, crônica ou cíclica); consequências (gravidade da doença e seu impacto no funcionamento físico, psicológico e social); e controle/cura (possibilidade de curar ou controlar a doença) $)^{(6-7)}$.

O MSC pode ser aplicado em estudos com populações diferentes, como se observa em estudo com pais de crianças e adolescentes oncológicos sobre a percepção da doença e o tratamento de seus filhos, o que permite fundamentar melhores estratégias de intervenções ${ }^{(8)}$. Neste estudo, a aplicação do MSC prevê que portadores de doenças como o DM respondam a uma doença em particular, com base em suas interações com o meio social, experiências passadas e seus conhecimentos implícitos sobre os significados da doença. As diversas fontes de informação com que tem contato permitem que a pessoa construa o seu próprio conjunto de crenças sobre a doença e seu tratamento ${ }^{(9)}$.

Destaca-se a importância deste estudo como meio de contextualizar o ser diabético, suas vulnerabilidades e o impacto na vida do paciente em atendimento ambulatorial e hospitalar, permitindo, assim, uma atuação multidimensional no cuidado das DCNT de forma eficaz, minimizando complicações no decorrer da doença.

Com base nos conceitos do MSC, este estudo tem como objetivo analisar o estado de saúde e as dimensões relacionadas ao DM de pacientes hospitalizados e ambulatoriais de um hospital de ensino à luz do Modelo do Senso Comum.

\section{Método}

Trata-se de estudo exploratório, descritivo, com análise qualitativa dos dados à luz de conceitos do $\mathrm{MSC}^{(7)}$. A coleta dos dados foi realizada no Ambulatório de Endocrinologia e Unidades de Internação Clínica de um hospital de ensino, em São Paulo (SP), entre os meses de novembro e dezembro de 2019. Foram convidados a participar do estudo todos os paciente com diagnóstico de DM internados nas unidades de internação de clínica médica e os pacientes atendidos no ambulatório de endocrinologia da instituição. Excluíram-se os pacientes internados em investigação de DM. Os participantes integraram o estudo por conveniência e houve cinco recusas quanto à participação, devido à falta de tempo, não estar disposto ou sonolento, em alguns casos. A coleta de dados foi realizada com base em um roteiro com questões com características sociodemográficas e clínicas relacionadas ao DM e questões específicas baseadas nos domínios do MSC e na literatura especializada.

A coleta dos dados foi interrompida quando o conjunto de dados obtido nas entrevistas mostrou-se redundante e suficiente para responder aos objetivos do estudo, de acordo com a matriz estruturada, baseada no MSC ${ }^{(6-7)}$. Adotou-se a análise de conteúdo dos discursos em sua 
modalidade dedutiva, por meio da qual os pesquisadores podem testar categorias, conceitos, modelos ou hipóteses pré-existentes, baseadas em conceitos prévios, originários de uma determinada teoria, por exemplo. As análises indutiva e dedutiva dividem-se em três fases principais: preparação, organização e relato dos resul$\operatorname{tados}^{(10)}$. Na fase de preparação, leu-se cautelosamente o material proveniente dos discursos, destacando palavras, frases e expressões relacionadas ao objetivo proposto para o estudo. $\mathrm{Na}$ sequência, foi elaborada uma matriz estruturada, orientada pelas dimensões do MSC: identidade, causas, duração, consequências e controle ou cura $^{(6-7)}$. Seguindo-se esses passos, selecionou-se apenas os aspectos relacionados às dimensões do modelo extraídos dos depoimentos dos participantes e reunidos em categorias apresentadas nos resultados.

Para preservar a identidade dos participantes, optou-se pela utilização do sistema alfanumérico, por meio do codinome $\mathrm{P}$, relativo ao participante, seguido da numeração arábica, conforme a ordem de ocorrência das entrevistas (P1, P2, P3... P20).

O estudo respeitou os preceitos éticos da condução de pesquisas com seres humanos, de acordo com a Resolução n. 466/12 do Conselho Nacional de Saúde e obteve aprovação do Comitê de Ética em Pesquisa (CEP) da instituição envolvida, conforme Certificado de Apresentação de Apreciação Ética (CAAE) n. 18488319.6.0000.5479.

\section{Resultados}

Participaram do estudo 20 pacientes internados e em acompanhamento ambulatorial. Em relação às características da amostra, a média de idade foi de 60,4 anos (DP $\pm 9,6$ ), com Índice de Massa Corpórea (IMC) médio de $32.6 \mathrm{~kg} / \mathrm{m}^{2}$, predomínio do sexo masculino $(65,0 \%)$, casados (40\%), brancos (55\%), com fundamental completo e médio completo (30\% cada), profissionais liberais (35\%) e católicos (60\%).

O conteúdo dos depoimentos obtidos foram reunido em categorias, segundo as cinco dimensões do MSC, a saber: identidade - expressa por rótulos abstratos, como o nome da doença e seus sintomas associados; causas - fatores relacionados à origem da doença; duração - curso da doença (aguda, crônica ou cíclica); consequências - gravidade da doença e seu impacto no funcionamento físico, psicológico e social; e controle/cura - possibilidade de curar ou controlar a doença ${ }^{(6-7)}$.

\section{Identidade da doença}

O que é o DM, as representações influenciadas por crenças individuais que podem comprometer a aceitação da doença e o tratamento, os comportamentos e o enfrentamento da situação vivida. Excetuando-se dois participantes, que referiram não saber o que é o diabetes, os demais foram capazes de definir a doença, com base em seus conhecimentos e crenças.

Para dois dos participantes, o diabetes é um problema orgânico, relacionado ao funcionamento de um órgão em especial, o pâncreas:

Diabetes é um problema no pâncreas que interfere no meu corpo todo. (P2).

Diabetes é um problema de açúcar no sangue por conta da não produção de insulina pelo pâncreas. (P5).

Outros três, relacionaram a doença diretamente aos hábitos alimentares e às suas restrições. Descreveu-se a intolerância à glicose, referindo-se a ela como "açúcar":

Diabetes, intolerância a açúcar, não posso comer nada c/açúcar. (P4).

Alimentação fora de hora, comer de 3 em 3 horas, não pode comer nada doce, nada enterrado. (P8).

Não pode comer certas coisas que atrapalha. (P16).

\section{Causas da doença}

$\mathrm{Na}$ perspectiva de seus portadores, de uma maneira geral, os participantes mostraram conhecer algumas das causas de sua doença. Para a metade dos entrevistados $(n=10)$, o diabetes tem como principal causa os maus hábitos de vida, em particular, a ingestão exagerada de alimentos ricos em carboidratos simples, como observado nos depoimentos a seguir: 
Acho que os hábitos de vida, principalmente a alimentação. (P2).

O açúcar, a massa. (P3).

Eu acho que é o açúcar que comemos. (P4).

Alimentação errada: pizza, macarrão, batata assada. (P14).

Outros, referiram-se à "obesidade" (P9), às questões genéticas (P14) e ao sedentarismo (P5), como prováveis fatores que os levaram a desenvolver a doença. Um dos participantes citou que sua doença foi consequente do consumo excessivo de álcool.

Pancreatite e excesso de álcool. (P10).

\section{Duração da doença e descoberta do diagnóstico}

Seis depoentes disseram que não identificaram sintomas até se sentirem mal e procurarem o serviço de emergência. Dentre os principais sinais e sintomas descritos pelos demais, destacaram-se: a poliúria, a polidipsia e as infecções recorrentes.

Percebi quando tava trabalhando. Muito xixi, sede, dor nas pernas. (P1).

Urina, com espuma, infecção no estômago [Helicobacter pilory] e urinária, muita sede, fazendo muito xixi, amargo na boca e parece areia. (P4).

Outros três participantes citaram as alterações visuais, tontura e emagrecimento:

Comecei a perceber nas vistas, pra assistir televisão, atrapalhava. (P3).

Emagrecimento, sede excessiva. (P6)

Pico de diabetes, visão embaçada, tontura, sede e ir mui to ao banheiro. (P12).

\section{Consequências e tratamento da doença}

Quanto às representações sobre as consequências da doença, observa-se que estiveram diretamente ligadas ao seu controle. Para os participantes, o controle dos índices glicêmicos foi responsável por importantes mudanças no presente, as quais podem ser determinantes para evitar as consequências graves da doença:

Agora o diabetes está mais controlado mesmo com o pro-

blema no pé, mas antigamente me atrapalbou muito nas questões de alimentação controlada, libido na relação sexual, fraqueza, cansaço, e visão turva. Espero nunca voltar a descontrolar, pois interfere em tudo na vida. (P2).

Responsabilidade de cuidar, mudar a alimentação e os remédios. (P12).

Quanto ao presente, os participantes citaram o incômodo consequente à doença e ao seu tratamento:

Falta de memória e cansaço. (P9).

Vistas embaralhadas. (P8)

Serfurado três vezes ao dia. (P10).

A quantidade [alta] de remédios, pelo mal-estar da bipoglicemia e cansaços ocasionais. (P5).

Dois entrevistados citaram que já vivenciam, no presente, as consequências de não terem tomado as precauções necessárias para assegurar a sua sobrevida livre das complicações da doença. Tal constatação expressou certa desesperança quanto ao futuro:

Acho que o AVC foi pela diabete, meu rim também não é bom. Não espero nada pra frente. (P1).

Má qualidade de vida já apareceu, visão, rins. (P7).

Quando alta me deixa irritada, tenho complicações nos rins. (P11).

Outros dois entrevistados citaram não ter percebido nenhuma repercussão significativa da doença até o momento em que participaram do estudo; um deles justificou que convive com a doença há pouco tempo:

Não teve nenhuma [consequência] não, descobri faz 6 meses. (P3).

O outro referiu não acreditar que haverá algo grave, por não ter percebido sinais indicativos de potenciais complicações:

Não percebi nada, e acho que não terá nenbuma consequência. (P13).

\section{Controle/cura da doença}

Em relação às representações sobre o controle/cura da doença, os depoentes expressaram preocupações quanto aos efeitos esperados ou aqueles que podem ser prejudiciais, conforme depoimentos a seguir: 
Eu acho que o medicamento faz efeito na hora, mas depois eu sinto falta de alguma coisa. A insulina também. Seria bom se sempre fizesse efeito. (P1).

Tomo muito remédio e não é bom tomar muito não, porque é ruim pro corpo. (P3).

Embora os participantes avaliem que a doença está sob controle, graças a seus constantes esforços em cumprir o tratamento prescrito, o monitoramento da doença, por meio de glicômetro, pareceu incomodar dois participantes, pela dor causada pela perfuração nos dedos para a realização da checagem dos índices glicêmicos, como também a administração de insulina pela via parenteral, que implica em injeções subcutâneas diárias:

\footnotetext{
Ótimo [o tratamento], menos a insulina por ser injeção. (P12).

Não gosto das furadas no dedo e da insulina. (P15).

Acho bom, só não gosto das furadas o dia inteiro. (P17).

Não gosto da insulina, então preferi os medicamentos. Mas provável que agora tenho a necessidade de mudar, isso já me preocupa. (P2).
}

\section{Discussão}

Segundo o International Diabetes Federation (IDF), é importante destacar que o DM e outras doenças crônicas têm uma prevalência maior em pessoas maiores de 50 anos de idade e têm aumentado significativamente, sendo uma das doenças crônicas não transmissíveis que mais cresce, principalmente nos países em desenvolvimento ${ }^{(1)}$.

O método misto permite identificar a relação das características da amostra e relacioná-las ao método do MSC, podendo identificar pontos de vulnerabilidade do paciente diabético em relação à doença, causas, duração, consequências e cura, o que destaca a importância de intervenção junto ao grupo para melhor adequação do tratamento e resultados.

Estudo realizado com o objetivo de avaliar o conhecimento sobre o diabetes, a atitude para o autocuidado e os fatores associados em idosos assistidos na atenção primária de saúde identificou que a atitude para o autocuidado em relação ao DM diminui com o tempo de diagnóstico e, por ser uma doença crônica, diminui a motivação dos pacientes para se envolver com o autocuidado, podendo também refletir em muitos sentimentos, como tristeza, medo, culpa e revolta, que necessitam ser superados para que se atinja a aceitação da doença e resulte em melhores resultados $^{(11)}$. Outro estudo aponta que ser homem, morar sozinho e ter alguma complicação do diabetes é fator associado a uma atitude negativa, relacionada ao autocuidado e complicações, que pode influenciar no enfrentamento da doença e no tratamento ${ }^{(12)}$.

A evolução da incidência das DCNT, principalmente o DM, está associada ao processo de envelhecimento e aos hábitos poucos saudáveis de vida, frequentemente observados na população brasileira. Associado a isso, encontra-se a transição demográfica e a necessidade de os profissionais da saúde buscarem desenvolver um planejamento estratégico, com programas de atendimentos específicos para avaliar as comorbidades e as complicações, que agregado às características sociodemográficas podem promover melhor controle da doença ${ }^{(13-14)}$.

No que se refere à identidade da doença, as falas de dois pacientes descrevem seu desconhecimento em relação à doença, diferente dos demais participantes que conseguem definir o DM baseado em seus conhecimentos e crenças, inclusive como um problema fisiológico que compromete o funcionamento do pâncreas.

Segundo a Diretriz Brasileira de Diabetes ${ }^{(15)}$, o DM atinge 180 milhões de pessoas no mundo. Com o aumento da obesidade e do sedentarismo, estima-se que, em 2025, mais de 300 milhões de pessoas venham a ter diabetes.

A expectativa de aumento da doença para os países em desenvolvimento é de 170\% (de 84 milhões para 228 milhões de pessoas afetadas). Essa projeção implica em um aparecimento do DM de modo mais precoce na população, quando comparado a outros países desenvolvidos ${ }^{(16)}$.

A causa real do DM deve ser esclarecida, para que o paciente tenha uma visão das consequências que a doença pode apresentar, bem como os fatores que influenciam para o agravo e complicações da doença. O DM hoje é considerado 
como problema de saúde pública e uma doença responsável por uma das maiores causas de mortalidade do mundo ${ }^{(17)}$.

A causa, na perspectiva de seus portadores, reflete fatores relacionados a maus hábitos de vida, questão genética e estilo de vida.

Estudo destaca que o conhecimento sobre o DM é insuficiente em muitos pacientes com diagnóstico confirmado da doença, principalmente quanto à procedência e como ela age no $\operatorname{organismo}^{(6)}$.

A alimentação inadequada é um dos fatores que potencializam a evolução e os agravos da doença, e os pacientes devem ser bem orientados sobre seu comportamento diante disso. A Sociedade Brasileira de Diabetes (SBD) recomenda uma alimentação adequada de alimentos rica em fibras, legumes, leguminosas e laticínios desnatados, para que favoreça o controle pós-prandial da glicemia, sensibilizando assim a ação da insulina ${ }^{(15)}$.

Em relação à questão social, estudos comprovaram que pacientes inseridos em programas sociais, como a Estratégia Saúde da Família (ESF), estão mais propensos a melhor controle glicêmico $^{(18)}$.

Quanto à duração da doença e descoberta do diagnóstico, alguns dos depoentes afirmaram não ter identificado sintomas até que estes surgiram subitamente e necessitaram de atendimento de Emergência, destacando sintomas clássicos da DM, como poliúria, polidipsia, infecções repetidas, alterações visuais, tontura e emagrecimento.

Os achados vão ao encontro dos resultados de um estudo que destaca a dificuldade de buscar informações sobre o aspecto negativo que a doença pode trazer para o paciente e seus familiares e o conhecimento prévio sobre a doença, interferindo na adesão ao tratamento ${ }^{(8)}$.

Por ser uma doença silenciosa, e muitas vezes assintomática, o que corrobora a fala dos entrevistados, a doença, muitas vezes, não é considerada importante por parte da população, o que dificulta a prevenção de complicações da doença. A OMS, em seu relatório, aponta que no ano de 2016 houve um crescimento acelerado da doença em países em desenvolvimento, o que levou a um aconselhamento para a realização de sensibilização da população, com práticas preventivas e educativas, a fim de paralisar o seu crescimento exacerbado ${ }^{(2)}$.

A literatura aponta para a relação das complicações relacionadas ao tempo da doença. Há evidências de que o maior número de anos vividos com DM pode ser afetado, não somente pela condição clínica, mas também pelo tratamento a que foi submetido ao longo da vida. Entretanto, essa afirmação pode ser distinta em diferentes países do mundo ${ }^{(19)}$.

Com base nos depoimentos dos participantes referente às representações sobre as consequências da doença, nota-se que a percepção dos depoentes está ligada ao controle da doença pela medição da glicemia, e, na opinião deles, sua inadequação está relacionada às complicações, sensações que geram incômodos, cansaço, alteração visual e o aumento do consumo de medicamentos, além das complicações, como a hipoglicemia. No entanto, destaca-se que alguns discursos estão associados ao fato da negligência no controle da doença, o que gera um sentimento de desesperança em relação à evolução e desfecho do DM.

Estudo que utilizou o modelo MSC com crianças e adolescentes oncológicos apontam para resultados semelhantes ao deste estudo, mesmo em condições fisiopatológicas distintas. Destaca-se a falta de ligação causal efetivamente em relação à doença, e o não pensar nessa relação de causa e efeito pode ser atribuída à condição do diagnóstico já causar estresse e sofrimento ao paciente e seus familiares ${ }^{(8)}$.

Outro sentimento de percepção das consequências do DM aponta para uma situação bastante preocupante, que é a subestimação das complicações da doença, principalmente por aqueles com recém-diagnóstico, ou simplesmente por não acreditar nelas.

As complicações relacionadas ao DM são a hipoglicemia, a CAD e o EHH. A hiperglicemia contribui para complicações macrovasculares que causam a doença cerebrovascular, doença arterial coronariana (DAC) e doença vascular 
periférica (DVP), enquanto as microvasculares causam nefropatias, neuropatias e retinopatia. $\mathrm{O}$ tratamento do diabetes deve ser iniciado imediatamente após o diagnóstico, para prevenção das complicações. Em relação a retinopatia, é importante monitorá-la mediante tratamento oftalmológico periódico, pois o paciente diabético tem 30 vezes mais possibilidades de ficar cego em relação ao não diabético ${ }^{(15)}$.

A perda visual ocorre de forma secundária à retinopatia diabética e está associada a uma diminuição significativa da qualidade de vida do paciente diabético, alterando sua dinâmica de vida diária, na maioria das vezes ${ }^{(20)}$.

Estudo realizado descreve que a diferença entre o desconhecimento da doença e o nível de escolaridade dos pacientes são tendências a não valorização das ações preventivas, subestimando a gravidade da doença e postergando a procura da assistência médica, o que dificulta a adesão ao tratamento e propicia o aumento das complicações $^{(21)}$.

No que diz respeito ao controle das representações sobre o controle/cura da doença, os depoentes descrevem preocupação quanto à forma de tratamento.

A adesão ao regime de tratamento do DM exige frequentes alterações no estilo de vida em diferentes áreas, como alimentação, medicação e atividade física, com implicações diretas na sua rotina diária. A não adesão ao regime terapêutico contribui para o descontrole da doença, sendo um desafio constante para o paciente e para a equipe de saúde ${ }^{(22)}$.

É fato que a prevenção da doença implica em reorganização significativa na atenção à saúde. No caso do DM, envolve prevenção do início dos sintomas (prevenção primária), prevenção de suas complicações agudas e crônicas (prevenção secundária) e controle das incapacidades produzidas pelas suas complicações (prevenção terciária) $^{(23-24)}$.

Uma estratégia de intervenção educativa com foco na prevenção é baseada nas medidas de autocuidado para o indivíduo com DM. Destaca-se que isso é fundamental, porém, nem todos têm acesso ou assimilam as informações fornecidas.
A literatura destaca que muitos dos profissionais de saúde não o fazem de modo adequado, por deficiência dos serviços de saúde ${ }^{(25)}$. As orientações são provenientes principalmente do enfermeiro, que, com base na anamnese e no exame físico, estabelece um planejamento individualizado, podendo implementar medidas de controle e minimização das complicações decorrentes do DM.

As orientações para os pacientes com DM, independentemente do tempo de doença, são efetivas para fundamentar o conhecimento das representações da doença e seu impacto na vida diária, a importância do tratamento e principalmente identificar precocemente as complicações.

\section{Considerações finais}

As concepções do estado de saúde e suas dimensões relacionadas ao DM dos pacientes internados ou em atendimento ambulatorial, interpretadas à luz do MSC, descrevem percepção de uma doença com sinais físicos e com potencial de complicação, inclusive associada à morte. Os relatos apontam para um desconhecimento das causas reais e das consequências que a doença pode trazer. É fato que ter o diagnóstico de DM reflete na QV dos pacientes, direta ou indiretamente, mesmo que os pacientes não a percebam no início da doença e negligenciem sinais de complicação.

Dessa forma, destaca-se a importância do papel dos profissionais de saúde na abordagem individualizada, de acordo com as particularidades da doença, como meio de implementar medidas de controle, educação em saúde e acompanhamento das necessidades individuais de cada paciente, hospitalizados ou em atendimento ambulatorial.

\section{Colaborações:}

1 - concepção, projeto, análise e interpretação dos dados: Luciana Soares Costa Santos e Alessandra Thomaz de Andrade; 
2 - redação do artigo e revisão crítica relevante do conteúdo intelectual: Luciana Soares Costa Santos, Fernanda Machado Silva Rodrigues e Lívia Keismanas de Ávila;

3 - aprovação final da versão a ser publicada: Luciana Soares Costa Santos, Fernanda Machado Silva Rodrigues e Lívia Keismanas de Ávila.

\section{Referências}

1. International Diabetes Federation. IDF Diabetes Atlas [Internet] 9th ed. Bruxelas (BE); 2019. [cited 2020 May 18]. Available from: https://www. diabetesatlas.org

2. World Health Organization. Global report on diabetes [Internet]. Genebra (CH); 2016 [cited 2019 Jun 27]. Available from: http://apps.who.int/iris/ bitstream/10665/204871/1/9789241565257_eng. pdf

3. Vilar L. Endocrinologia Clínica. 6a ed. Rio de Janeiro: Guanabara Koogan; 2016.

4. American Diabetes Association. Improving Care and Promotion Health in Populations: Standards of Medical Care in Diabetes-2019. Diabetes Care. 2019;42(Suppl 1):S7-S12. DOI: https://doi. org/10.2337/dc19-S001

5. Parsa P, Ahmadinia-Tabeshb R, Mohammadic Y, Khoramid $\mathrm{N}$. Investigating the relationship between quality of life with lipid and glucose levels in Iranian diabetic patients. Diabetes Metab Syndr. 2017;11(Suppl 2):S879-S83. DOI: https:// doi.org/10.1016/j.dsx.2017.07.009

6. Leventhal $\mathrm{H}$, Brissette $\mathrm{I}$, Leventhal EA. The common-sense model of self-regulation of health and illness. In: Cameron LD, Leventhal $\mathrm{H}$, editores. The self-regulation of health and illness behavior. New York: Routledge; 2003. p. 42-65.

7. Fall E, Chakroun-Baggioni N, Böhme P, Maqdasy $S$, Izaute $M$, Tauveron I. Common sense model of self-regulation for understanding adherence and quality of life in type 2 diabetes with structural equation modeling. Patient Educ Couns. 2021;104(1):171-8. DOI: 10.1016/j. pec. 2020.06 .023

8. Silva-Rodrigues FM, Vulczak VLS, Alencar CF, Santos LSC, Nascimento LC. Concepções dos pais acerca da doença oncológica e do tratamento quimioterápico de seus filhos. Rev Enferm UFSM. 2020;10:e33. DOI:10.5902/2179769235898
9. Shiyanbola OO, Ward EC, Brown CM. Utilizing the common sense model to explore African Americans' perception of type 2 diabetes: a qualitative study. PloS One. 2018;13(11):e0207692. DOI: 10.1371/journal. pone. 0207692

10. Elo S, Kyngäs H. The qualitative content analysis process. J Adv Nurs. 2008;62(1):107-15. DOI: 10.1111/j.1365-2648.2007.04569.x

11. Sousa MC, Malaquias BSS, Chavaglia SRR, Ohl RIB, Paula FPS, Silva KS, et al. Autoeficácia em idosos com Diabetes Mellitus tipo 2. Rev Bras Enferm. 2020;73(3):e20180980. DOI: http://dx.doi.org/10.1590/0034-7167-2018-0980

12. Borba AKTOT, Arruda IKG, Marques APO, Leal MCC, Diniz AS. Conhecimento sobre O diabetes e atitude para o autocuidado de idosos na atenção primária à saúde. Ciênc saúde coletiva. 2019;24(1):125-36. DOI: http://dx.doi. org/10.1590/1413-81232018241.35052016

13. Kovacs R, Barreto JOM, Silva EN, Borghi J, Kristensen SR, Costa DRT, et al. Socioeconomic inequalities in the quality of primary care under Brazil's national pay-for-performance programme: a longitudinal study of family health teams. Lancet Glob Health. 2021;9(3):e331-e9. DOI: https://doi. org/10.1016/S2214-109X(20)30480-0

14. Marinho F, Passos VMA, Malta DC, França EB, Abreu DMX, Araújo VEM, et al. Burden of disease in Brazil, 1990-2016: a systematic subnational analysis for the Global Burden of Disease Study 2016. Lancet. 2016;392(10149):760-75. DOI: 10.1016/S0140-6736(18)31221-2

15. Forti AC, Pires AC, Pittito BA, Gerchman F, Oliveira JEP, Zajdenverg L, et al, organizadores e coordenadores. Diretrizes da Sociedade Brasileira de Diabetes 2019-2020 [Internet]. São Paulo: Sociedade Brasileira de Diabetes; 2019 [cited 2019 July 13]. Available from: https://www.diabetes. org.br/profissionais/images/DIRETRIZESCOMPLETA-2019-2020.pdf

16. King H, Aubert RE, Herman WH. Global burden of diabetes, 1995-2025: prevalence, numerical estimates, and projections. Diabetes Care. 1998;21(9):1414-31.DOI: 10.2337/diacare.21.9.1414

17. Boyle JP, Honeycutt AA, Narayan KM Hoerger TJ, Geiss LS, Chen H, et al. Projection of diabetes burden through 2050: impact of changing demography and disease prevalence in the U.S. Diabetes 
Care. 2001;24(11):1936-40. DOI: 10.2337/ diacare.24.11.1936

18. Diaz N, Moreira PB, Haluch RF, Ravazzani AC, Kusma SZ. O impacto do diabetes mellitus tipo 2 na qualidade de vida. Rev Med UFPR. 2016;3(1):5-12. DOI: 10.5380/rmu.v3i1.46380

19. Cortez DN, Reis IA, Souza DAS, Macedo MML, Torres HC. Complicações e o tempo de diagnóstico do diabetes mellitus na atenção primária. Acta paul enferm. 2015;28(3):250-5. DOI: http:// dx.doi.org/10.1590/1982-0194201500042

20. Brown MM, Brown GC, Sharma S, Shah G. Utility values and diabetic retinopathy. Am J Ophthalmol. 1999;128(3):324-30. DOI: https://doi. org/10.1016/S0002-9394(99)00146-4

21. Flor LS, Campos MR. The prevalence of diabetes mellitus and its associated factors in the Brazilian adult population: evidence from a population-based survey. Rev bras epidemiol. 2017;20(1):16-29. DOI: http://dx.doi.org/10.1590/ 1980-5497201700010002

22. Silva I, Pais-Ribeiro J, Cardoso H. Adesão ao tratamento da diabetes Mellitus: A importância das características demográficas e clínicas. Referência [Internet]. 2006 [cited 2019 Oct 20];
2(2):34-41. Available from: https://www.redalyc. org/pdf/3882/388242124002.pdf

23. Costa ML, Moraes RB, Vaz DWN, Santos GA, Duarte RCC, Santos Júnior JAB, et al. Avaliação dos pacientes com Diabetes e Hipertensão em uma Estratégia de Saúde da Família localizada na zona rural do interior do Estado do Pará. Res Soc Develop. 2021;10(3):e2610313025. DOI: 10.33448/ rsd-v10i3.13025

24. Girotto E, Andrade SM, Cabrera MAS, Matsuo T. Adesão ao tratamento farmacológico e não farmacológico e fatores associados na atenção primária da hipertensão arterial. Ciênc saúde coletiva. 2013;18(6):1763-72. DOI: http://dx.doi. org/10.1590/S1413-81232013000600027

25. Andrade NHS, Sasso-Mendes KD, Faria HTG, Martins TA, Santos MA, Teixeira CRS, et al. Pacientes com Diabetes Mellitus: Cuidados e Prevenção de Pé Diabéticos em Atenção Primária à Saúde. Rev enferm UERJ [Internet]. 2010 [cited 2019 Oct 20];18(4):612-21. Available from: http:// www.revenf.bvs.br/pdf/reuerj/v18n4/v18n4a19. pdf

Recebido: 16 de outubro de 2020

Aprovado: 29 de março de 2021

Publicado: 26 de abril de 2021

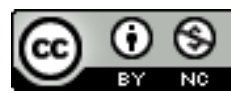

A Revista Baiana de Enfermagem utiliza a Licença Creative Commons - Atribuição-NãoComercial 4.0 Internacional.

https://creativecommons.org/licenses/by-nc/4.0/

Este artigo é de acesso aberto distribuído sob os termos da Licença Creative Commons (CC BY-NC).

Esta licença permite que outros remixem, adaptem e criem a partir do seu trabalho para fins não comerciais. Embora os novos trabalhos tenham de lhe atribuir o devido crédito e não possam ser usados para fins comerciais, os usuários não têm de licenciar esses trabalhos derivados sob os mesmos termos. 